\section{Low Incidence of Clostridium difficile Infection (CDI) in Patients Treated with Outpatient Parenteral Antimicrobial Therapy (OPAT)}

To the Editor-Outpatient parenteral antimicrobial therapy (OPAT) is a safe, clinically effective, convenient and cost-effective way of administering parenteral antimicrobials while allowing the patient to reside in the community. ${ }^{1,2}$ It has been hypothesized that OPAT reduces the incidence of hospital-acquired infections by cutting down the length of stay and exposure to the healthcare facility. ${ }^{3}$ However, there are very few studies on the incidence and outcomes of OPAT in relation to C. difficile infection (CDI). Two previous European studies have reported $<1 \%$ incidence of CDI in their OPAT population. ${ }^{3,4} \mathrm{~A}$ recent questionnaire survey of infectious diseases (ID) physicians conducted by the Infectious Diseases Society of America (IDSA), reported that the incidence of CDI in the OPAT population may be higher than that reported in the European studies. ${ }^{5}$ With increasing reports of community onset/acquired CDI with minimal or no exposure to a healthcare facility, ${ }^{6}$ it is possible that OPAT patients on broad-spectrum antibiotics are at increased risk of CDI.

Our institution has a large and well-organized OPAT program in which all antimicrobial management is supervised by infectious disease staff physicians, and infectious disease followup arrangements are made before patients are discharged from hospital. ${ }^{2}$ This arrangement provided the opportunity to examine the incidence and outcomes of CDI in our OPAT cohort. The study protocol was approved by the Institutional Review Board of Cleveland Clinic. We retrospectively reviewed all adult patients discharged home on OPAT from January 1, 2013, to January 1, 2014, whose OPAT medications were provided by the Cleveland Clinic Home Infusion Pharmacy (CCHIP). Community-onset, healthcare facility-associated (CO-HCFA) CDI was defined as diarrheal symptoms (watery diarrhea $>3$ times per day) and positive stool toxin-B polymerase chain reaction (PCR) for Clostridium difficile, in the community or within the first 3 days of readmission, provided the diagnosis was made less than 4 weeks since discharge from a healthcare facility. CDI severity was defined per the Infectious Disease Society of America (IDSA) ${ }^{7}$; recurrence was defined as symptomatic diarrhea with positivestool PCR within 8 weeks of successful treatment of the previous episode. In patients with multiple OPAT courses, only the initial OPAT course was included.

During the study period, a total of 1,462 patients received one or more courses of OPAT at home. Of these, 681 patients received medications for at least 1 OPAT course via the Cleveland Clinic Home Infusion Pharmacy. Five of these 681 patients $(0.73 \%)$ developed CO-HCFA CDI with an incidence rate of 0.26 cases per 1000 patient-days. Table 1 summarizes the demographic characteristics of and outcomes for these 5 patients. The most frequently administered antimicrobials were pipercillin/tazobactam and amikacin, with a median duration of 12 days (interquartile range [IQR]: 6.5-28). Four of these 5 patients completed OPAT prior to developing CO-HCFA CDI. The median period between OPAT completion to developing CDI was 9.5 days (IQR: $3-13$ ). Prior to developing CDI, each of the 5 patients had direct exposure to a healthcare facility that was more than a simple follow-up office visit after discharge from the hospital. The median duration from healthcare facility exposure to development of CDI was 8 days (IQR: $2-11.5$ ). Of these 5 patients, 2 had hospital readmissions (non-CDI related) and 3 had outpatient procedures. Four of these 5 patients $(80 \%)$ were on concomitant acid-suppressive therapy. All patients had mild-to-moderate CDI and responded well to medical therapy. None of the 5 patients had a recurrent $\mathrm{CDI}$, and there was no attributable 90-day mortality.

In this large study of 681 OPAT patients, CO-HCFA CDI was uncommon, occurring in $<1 \%$ of patients and at an incidence rate of less than 1 per 1000 person-days. None of the patients developed severe CDI; CDI did not progress to recurrent CDI; and there were no CDI-related readmissions. All CO-HCFA CDI patients had an institutional healthcare exposure, and $80 \%$ of people who developed CDI were on concomitant acid-suppression therapy.

Our findings on the incidence of CDI are in agreement with that reported in the two European studies. ${ }^{3,4}$ The two major risk factors for developing CDI are antimicrobial therapy and healthcare facility exposure. ${ }^{7}$ Four of the 5 patients in our study developed CDI with 10 days of completing their antibiotic OPAT medications and all five patients had a recent healthcare facility exposure other than their regular follow up office visits, after they were discharged from hospital and before the development of CDI. Both inpatient and outpatient healthcare exposure have been attributed to increased risk of CDI development. ${ }^{8,9}$ Therefore, antimicrobial stewardship and effective infection control measures will play an important role in further reducing the incidence of CDI in this population. Four of the 5 patients in our study were on concomitant acid-suppressive therapy; a few recent meta-analyses have suggested an association between proton-pump inhibitor (PPI) exposure and risk of developing CDI. ${ }^{10}$ This underscores the need for more judicious use of this class of medications.

Our study has some inherent limitations. The major limitation is that it is based on retrospective observational data. CDIs that were diagnosed based on tests done outside the Cleveland Clinic Health System were not identified. The small number of CDIs precludes an analysis for risk factors associated with development of CDIs in OPAT patients. Finally, our study is a single-center experience, and additional studies are needed in other settings. 


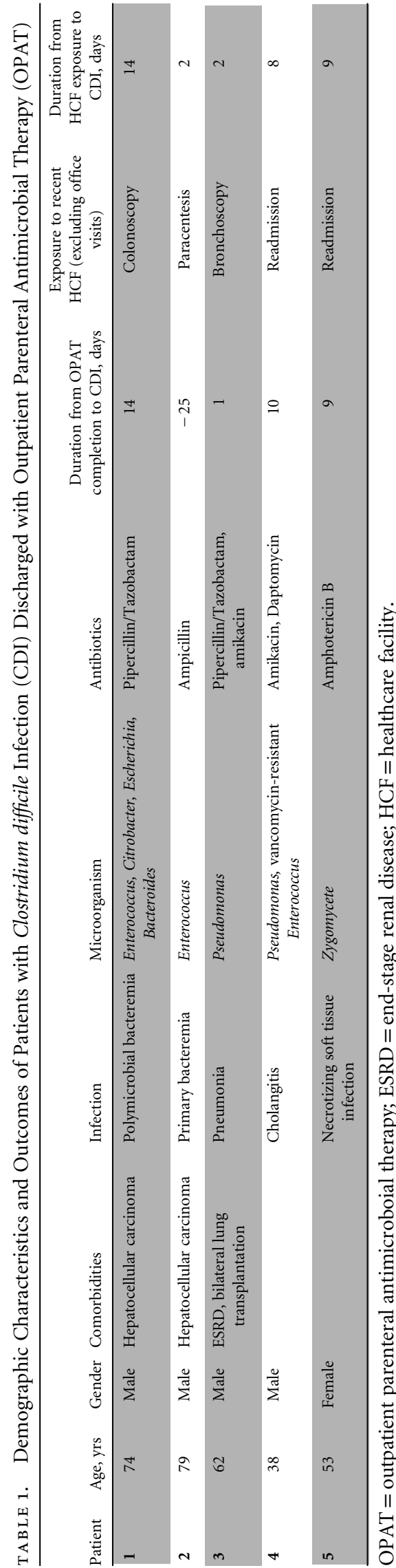

Overall, this study showed that CO-HCFA CDI is uncommon in the OPAT population. This helps physicians weigh the risks and benefits of OPAT and emphasizes the need for continued follow-up beyond the termination of antibiotic therapy.

\section{ACKNOWLEDGMENTS}

Financial support. None reported.

Potential conflicts of interest. All authors report no conflicts of interest relevant to this article.

\section{Ken K. Wong, MD; ${ }^{1}$ \\ Thomas G. Fraser, MD; ${ }^{1}$ Nabin K. Shrestha, MD, MPH; ${ }^{1}$ Cynthia Fatica, $\mathrm{RN}^{3}$ Abhishek Deshpande, $\mathrm{MD}, \mathrm{PhD}^{1,2}$}

Affiliations: 1. Department of Infectious Diseases, Medicine Institute, Cleveland Clinic, Cleveland, $\mathrm{OH} 44195 ; 2$. Medicine Institute Center for Value Based Care Research, Cleveland Clinic, Cleveland, OH 44195; 3. Department of Infection Prevention, Quality and Patient Safety Institute, Cleveland Clinic, Cleveland, OH 44195.

Address correspondence to Abhishek Deshpande, MD, $\mathrm{PhD}$, Medicine Institute Center for Value Based Care Research, 9500 Euclid Avenue, Desk G10, Cleveland Clinic, Cleveland, OH 44195 (abhishekdp@gmail.com). Infect Control Hosp Epidemiol 2015;36(1):110-112 (C) 2015 by The Society for Healthcare Epidemiology of America. All rights reserved. 0899-823X/2015/3601-0016. DOI: 10.1017/ice.2014.6

\section{REFERENCES}

1. Tice AD, Rehm SJ, Dalovisio JR, et al. Practice guidelines for outpatient parenteral antimicrobial therapy. IDSA guidelines. Clin Infect Dis 2004;38:1651-1672.

2. Gordon SM, Shrestha NK, Rehm SJ. Transitioning antimicrobial stewardship beyond the hospital: the cleveland clinic's community-based parenteral anti-infective therapy (CoPAT) program. J Hosp Med 2011;6 Suppl 1:S24-S30.

3. Chapman AL, Dixon S, Andrews D, Lillie PJ, Bazaz R, Patchett JD. Clinical efficacy and cost-effectiveness of outpatient parenteral antibiotic therapy (OPAT): A UK perspective. $J$ Antimicrob Chemother 2009;64:1316-1324.

4. Barr DA, Semple L, Seaton RA. Outpatient parenteral antimicrobial therapy (OPAT) in a teaching hospital-based practice: a retrospective cohort study describing experience and evolution over 10 years. Int J Antimicrob Agents 2012;39:407-413.

5. Lane MA, Marschall J, Beekmann SE, et al. Outpatient parenteral antimicrobial therapy practices among adult infectious disease physicians. Infect Control Hosp Epidemiol 2014;35:839-844.

6. Kutty PK, Woods CW, Sena AC, et al. Risk factors for and estimated incidence of community-associated Clostridium difficile infection, North Carolina, USA. Emerg Infect Dis 2010;16:197-204.

7. Cohen SH, Gerding DN, Johnson S, et al. Clinical practice guidelines for clostridium difficile infection in adults: 2010 update by the society for healthcare epidemiology of america (SHEA) and the infectious diseases society of america (IDSA). Infect Control Hosp Epidemiol 2010;31:431-455.

8. Samore MH, DeGirolami PC, Tlucko A, Lichtenberg DA, Melvin ZA, Karchmer AW. Clostridium difficile colonization and diarrhea at a tertiary care hospital. Clin Infect Dis 1994;18:181-187. 
9. Jury LA, Sitzlar B, Kundrapu S, et al. Outpatient healthcare settings and transmission of clostridium difficile. PLoS One 2013;8:e70175.

10. Deshpande A, Pant C, Pasupuleti V, et al. Association between proton pump inhibitor therapy and clostridium difficile infection in a meta-analysis. Clin Gastroenterol Hepatol 2012;10: 225-233.

\section{Code Flash: How an Interdisciplinary Team Eradicated Immediate-Use Steam Sterilization}

Previous Presentations: This information was presented at the Arkansas Nurses Association with preliminary data in October of 2012.

To the Editor-The 2014 Joint Commission National Patient Safety Goals include the prevention of infections following surgery. One potential cause of surgical site infection (SSI) is the use of reusable medical equipment that has undergone flash sterilization, also known as immediate-use steam sterilization (IUSS). This rapid means of sterilization facilitates replacement of an instrument that is unexpectedly required or contaminated during a surgical case. However, the literature associates IUSS with adverse events for patients. An internal review of IUSS at our facility revealed that we exceeded the Veterans' Health Administration benchmark of 1\%.

The leadership at our facility chartered an interdisciplinary team tasked with decreasing the use of IUSS. The team proposed the development of a "fast track" method of sterilization to replace the perceived need to use the flash sterilizer in the operating room. This conceptualization encountered resistance from surgeons and surgery staff who believed IUSS of contaminated instruments provided the only option for avoiding unwanted delays during surgery. Following a crucial conversation, the Chief of Surgery concurred with the implementation of a new system provided that the entire process required less than 30 minutes.

The team named this innovative system "Code Flash," building from the sense of urgency associated with the word "Code" (eg, "Code Blue" for cardiopulmonary arrest) and memories of flash sterilization associated with the word "Flash." This word selection process emphasized the replacement of old processes with a new one. Code Flash is the process of emergently transporting the needed surgical equipment to Sterile Processing Services, where it is reprocessed and returned to the OR to eliminate the need for IUSS. The staff initiates a Code Flash process when an instrument is contaminated or if an unanticipated need for an instrument arises.

The OR staff notifies SPS immediately via the Code Flash pager or telephone, specifying the needed instrument and the transport method that is to be used. SPS sends a Code Flash runner to the OR to obtain the instrument or the runner awaits the arrival of the instrument via a dedicated dumbwaiter. Meanwhile, a member of the SPS staff (with OR staff available for clarification) searches a database for the availability and location of a duplicate instrument available for use. If located, the Code Flash SPS staff member retrieves the instrument, delivers it directly to the OR suite, and places it in the hands of the OR scrub nurse or physician. If a replacement instrument is not available or is being reprocessed, SPS continues reprocessing the contaminated instrument as well. During a Code Flash, the dedicated SPS runner hand carries the instrument throughout the reprocessing area, eliminating the potential for a misplaced instrument, and continually communicates the emergent need for the instrument. With Code Flash, the reprocessing of the contaminated instrument and the search for a replacement instrument occur simultaneously.

To facilitate accurate communication, the team has standardized a list of more than 5,000 different instrument types and 1,000 instrument sets. Doing so has improved the efficiency of the search process significantly. Removing duplications, slang terminology, and misspelled entries has expedited the SPS staff's ability to determine whether a duplicate instrument is available. Rapid location of the instrument with the support of the revamped database facilitates delivery of a replacement instrument while the contaminated instrument continues the Code Flash sterilization process through the dedicated sterilizer. This clarity of communication has significantly improved the flow of Code Flash activities as well as physician, nurse, and staff satisfaction with the process.

Notably, the initial decrease in IUSS was encouraged by careful contemplation prior to using flash sterilization: we required staff to brief every IUSS to facility leadership. Prior to the implementation of Code Flash, IUSS rates varied from $1.5 \%$ to $5.5 \%$ ( 25 to 50 uses per month), and process implementation suffered from significant variance. Following the implementation of Code Flash, IUSS dropped immediately, and we have sustained the virtual elimination of IUSS for almost 3 years. Currently, our facility has not used IUSS since the second quarter of FY 2014 (Figure 1).

Code Flash has exerted multidisciplinary impact; it is discussed throughout all levels of the facility and has become a part of the culture of the organization. Our staff experiences the impact of Code Flash in the increased efficiency of reprocessing contaminated instruments resulting from this innovative standardized procedure for rapid replacement of urgently needed instruments.

With Code Flash, the redesign team has addressed the leading reasons for IUSS, specifically, communication issues and item contamination during a procedure, while increasing the efficiency of surgical instrument sterilization in our facility. By demonstrating that an instrument with superior decontamination can be returned to the OR more efficiently, surgical staff no longer perceive a need for flash sterilization. In conjunction with this procedural change, the team has addressed 